\title{
Simulation and Visualization of the electrical Activity of the Heart with focal ventricular tachycardia in a 3D Model
}

Christian Quester, Biomedical Engineering Department of University of Applied Sciences Offenburg, Badstrasse 24, D77652 Offenburg, Germany, e-mail: cquester@ stud.hs-offenburg.de

Matthias Heinke, Peter Osypka Institute of Medical Engineering, Departement of Electrical Engineering, Medical Engineering \& Computer Science, University of Applied Sciences Offenburg, Badstrasse 24, D-77652 Offenburg, Germany, e-mail: matthias.heinke@ @s-offenburg.de

Johannes Hörth, Peter Osypka Institute of Medical Engineering, Departement of Electrical Engineering, Medical Engineering \& Computer Science, University of Applied Sciences Offenburg, Badstrasse 24, D-77652 Offenburg, Germany, e-mail: Johannes.hoerth@hs-offenburg.de

\section{Background}

Patients with focal ventricular tachycardia are at risk of hemodynamic failure and if no treatment is provided the mortality rate can exceed $30 \%$. Therefore, medical professionals must be adequately trained in the management of these conditions. To achieve the best treatment, the origin of the abnormality should be known, as well as the course of the disease. This study provides an opportunity to visualize various focal ventricular tachycardias using the Offenburg cardiac rhythm model.

\section{Methods}

Modeling and simulation of focal ventricular tachycardias in the Offenburg heart rhythm model was performed using CST (Computer Simulation Technology) software from Dessault Systèms. A bundle of nerve tissue in different regions in the left and right ventricle was defined as the focus in the already existing heart rhythm model. This ultimately served as the origin of the focal excitation sites. For the simulations, the heart rhythm model was divided into a mesh consisting of 5354516 tetrahedra, which is required to calculate the electric field lines.

\section{Results}

The simulations in the Offenburg heart rhythm model were able to successfully represent the progression of focal ventricular tachycardia in the heart using measured electrical field lines. The simulation results were realized as an animated sequence of images running in real time at a frame rate of 20 frames per second. By changing the frame rate, these simulations can additionally be produced at different speeds.

\section{Conclusion}

The Offenburg cardiac rhythm model allows visualization of focal ventricular arrhythmias using computer simulations. By selecting the frame rate, the speed of the simulation results can be adjusted accordingly to visualize the electric field lines of focal ventricular tachycardias in more detail. The static and dynamic simulation results could be used in the future for teaching and research, including the training of medical professionals. 